\title{
Negative pathology of ureteral carcinoma significantly delaying the diagnosis of the primary tumor of osteoblastic metastases: A case report and review of the literature
}

\author{
YA-LAN WU ${ }^{1-3^{*}}$, YONG-MEI LIU ${ }^{2,3^{*}}$, HUI-JIAO CHEN ${ }^{4}$, YONG-SHENG WANG $^{2,3}$ and YOU LU ${ }^{2,3}$ \\ ${ }^{1}$ Department of Oncology, Chengdu Shang Jin Nan Fu Hospital; ${ }^{2}$ Department of Thoracic Oncology, \\ Cancer Center; ${ }^{3}$ State Key Laboratory of Biotherapy; ${ }^{4}$ Department of Pathology, West China Hospital, \\ West China Medical School, Sichuan University, Chengdu, Sichuan 610041, P.R. China
}

Received April 7, 2015; Accepted June 29, 2016

DOI: $10.3892 / \mathrm{ol} .2016 .5023$

\begin{abstract}
Ureteral urothelial carcinoma (UC) is a rare malignant tumor. The most common clinical manifestations of ureteral UC are hematuria, increased urinary frequency, dysuria and pain. The diagnosis of ureteral UC is made via radiography, endoscopy and pathology. Although osteoblastic destruction is usually observed in metastasis of prostate cancer, UC can also be a reason for osteoblastic metastasis. The present study reports the case of a 66-year-old man presenting with osteoblastic metastases, in which the primary tumor was finally diagnosed as a ureteral UC. However, the lack of pathological evidence significantly delayed the diagnosis of the primary tumor ( $>6$ months), even though the results of radiographic examination, and the type and mode of bone metastases significantly suggested a ureteral UC. The case reveals that a suitable screening test should be recommended for patients at high risk due to the possibility of a negative pathology result for ureteral UC. Additionally, a more efficient diagnostic method is required. Moreover, the possibility of new diagnostic criterion that do not rely on the pathology of primary foci in ureteral UC should be considered in future.
\end{abstract}

\section{Introduction}

Urothelial carcinoma (UC) is a common tumor that is identified most frequently in patients aged $50-80$ years, and which

Correspondence to: Professor You Lu or Dr Ya-Lan Wu, Department of Thoracic Oncology, Cancer Center, West China Hospital, West China Medical School, Sichuan University, 37 Guoxue Lane, Chengdu, Sichuan 610041, P.R. China

E-mail:radyoulu@hotmail.com

E-mail: croweggwu@outlook.com

*Contributed equally

Key words: ureteral urothelial carcinoma, diagnostic method, diagnostic criterion, screening test has a 2:1 male predominance (1). UC can be located in the bladder, renal pelvis or ureter with a relative frequency of 50:3:1 (2). The natural history of upper tract urothelial carcinoma (UTUC) differs from that of bladder cancer; $60 \%$ of UTUCs are invasive at diagnosis compared with only $15-25 \%$ of bladder tumors $(3,4)$. The majority of UCs are detected in the early stage, such that the patients often show long-term survival (5). For metastatic UC, systemic chemotherapy is recommended (5).

Ureteral UC is a rare malignant tumor, which accounts for $\sim 6 \%$ of all tumors of the upper urinary tract (1). The diagnosis of ureteral UC is made via radiography, endoscopy and pathology. Urinary obstruction is one of the typical imaging features (2). Although osteoblastic destruction is observed in the metastasis of prostate cancer, UC can also be the reason for osteoblastic metastasis. The treatment for metastatic ureteral $\mathrm{UC}$ is also systemic chemotherapy, and the same regimens used for bladder UC are recommended $(5,6)$. The present study reports the case of a 66-year-old male presenting with osteoblastic metastases, in which the primary tumor was finally diagnosed as a ureteral UC. However, the lack of pathological evidence significantly delayed the diagnosis of the primary tumor. Written informed consent was obtained from the patient's family.

\section{Case report}

A 66-year-old man presented to the Outpatient Department of the West China Hospital (Chengdu, Sichuan, China) on December 25, 2012 due to a 1-year history of thoracodorsal pain and a 2-week history of left-upper limb numbness. Positron emission tomography/computed tomography (CT) performed at another hospital on August 30, 2012 had revealed an increased bone density and fluorodeoxyglucose (FDG) metabolism of the pelvis and several vertebrae, increased FDG metabolism of enlarged lymph nodes inside the abdominopelvic cavity and a non-functioning right kidney (Fig. 1). Furthermore, enhanced magnetic resonance imaging of the abdominopelvic cavity had revealed several partly and unevenly enhanced nodules of the liver (the largest was $1.4 \mathrm{~cm}$ in diameter), and an unevenly enhanced stenotic right 
A
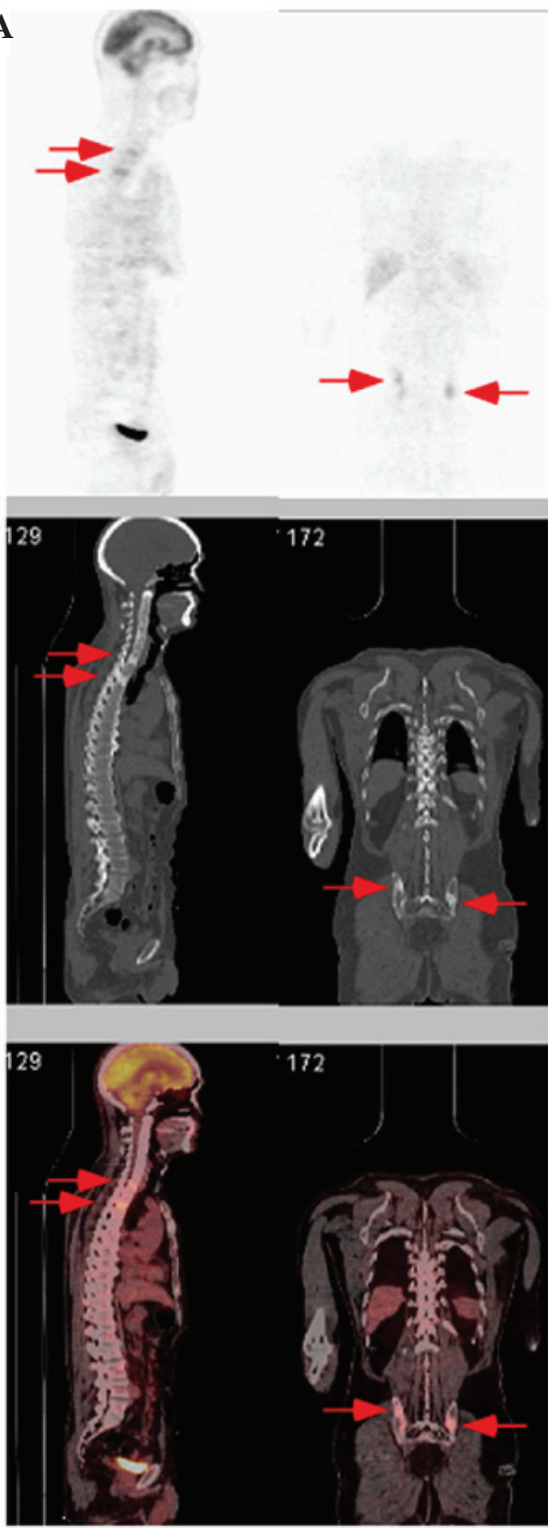

B
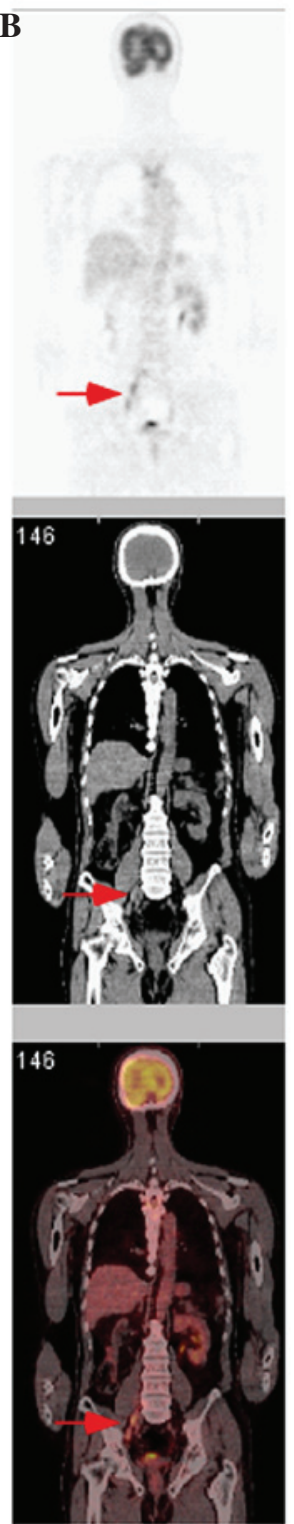

C
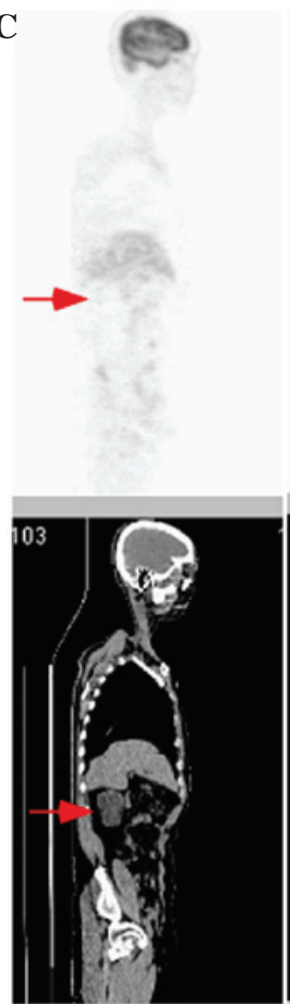

103

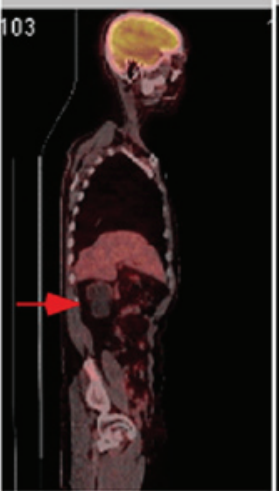

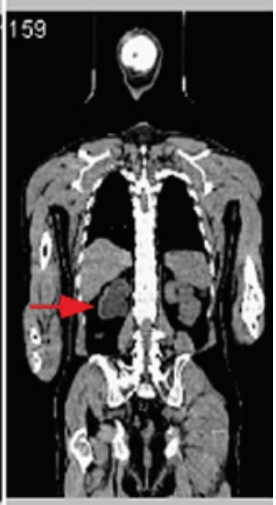
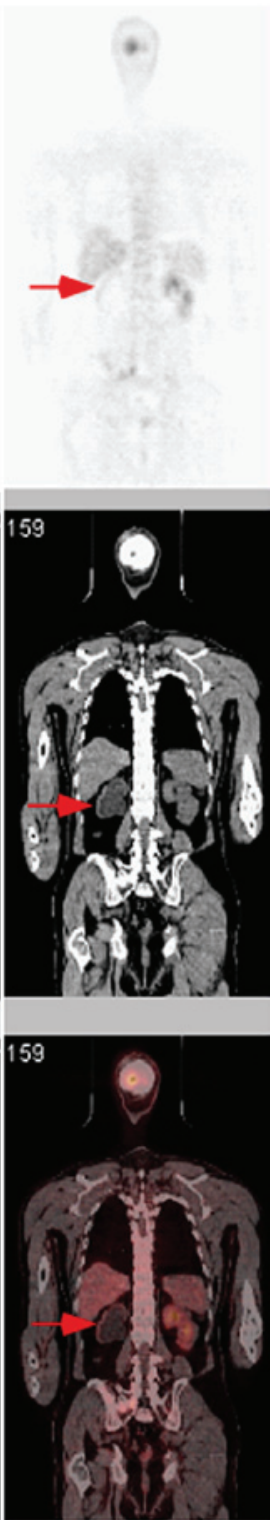

Figure 1. Results of positron emission tomography/computed tomography. (A) Increased bone density and fluorodeoxyglucose (FDG) metabolism of the pelvis and several vertebrae. (B) Increased FDG metabolism of several enlarged lymph nodes inside the abdominopelvic cavity. (C) The right kidney with dilated renal pelvis and atrophic renal parenchyma. Red arrows indicate the pathological foci.

ureter with a thickened wall. However, the ureteral endoscopy examination was negative, and pathological examinations of the prostate, bone marrow and voided urine had not detected any malignant cells.

In the Outpatient Operating Room of the West China Hospital, the patient received another multipoint biopsy of the prostate. However, a pathological examination of the prostatic biopsy, performed by the Department of Pathology at our hospital, found only low-grade prostatic intra-epithelial neoplasia and a few focal atypical glands. The patient then received another bone marrow biopsy (January 2,2013), and smears revealed a large number of atypical cell clusters. A flow cytometric analysis of these particular cells, performed on January 3, 2013, showed negative results for cluster of differentiation (CD)45, CD2, CD5, CD7, CD16, CD56, CD10, CD19, CD20, CD38, light-chain immunoglobulin, CD34, human leukocyte antigen-antigen D related and CD117. However, immunohistochemical staining performed by the Department of Pathology on January 14, 2013 showed positive results for pan cytokeratin (PCK), epithelial membrane antigen, CK7 and tumor protein 63 (p63), and negative results for CK5/6, CK20, thyroid transcription factor-1, prostate-specific antigen (PSA) and S-100 (Fig. 2A and B). Accordingly, the patient was diagnosed with malignant bone metastases, and the osteoblastic destruction was deemed not caused by prostate cancer.

The patient was hospitalized and administered drugs to control the pain $(90 \mathrm{mg}$ morphine hydrochloride sustained-release tablets every $12 \mathrm{~h}$ ) and nerve symptoms (thrice daily $0.3 \mathrm{~g}$ oral gabapentin and once daily $10 \mathrm{mg}$ intravenous dexamethasone for 4 days). The medical history revealed no family history of genetic diseases, but the patient was a retired employee of an oil company, therefore, contact was made with oil and various petroleum products during this time. To search for the primary tumor, the patient received thoracic and abdominal enhanced X-ray CT scans. The scans revealed liver metastases $(3.9 \times 3.0 \mathrm{~cm})$, and suggested 

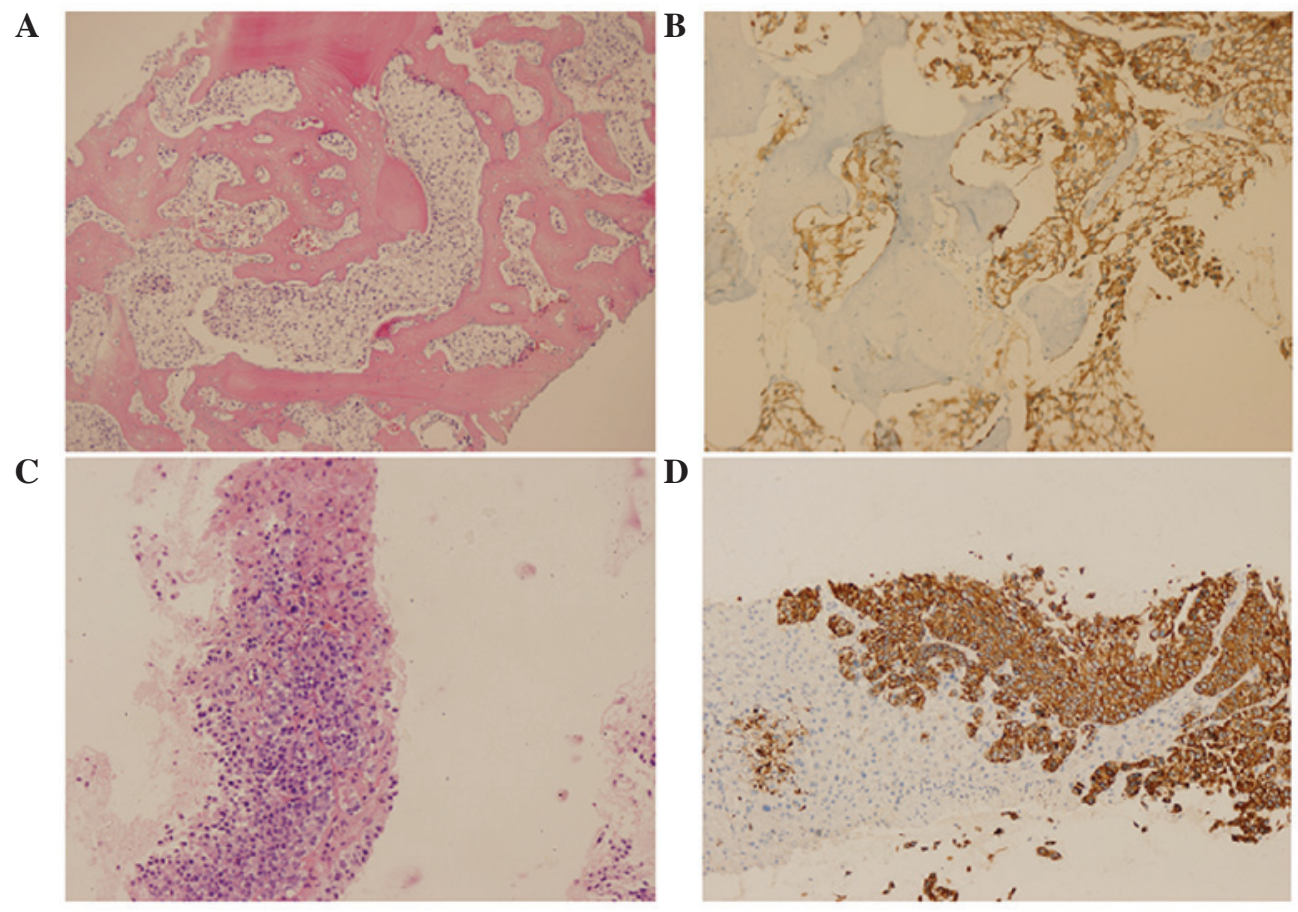

Figure 2. Pathological examinations of bone marrow and liver tissue. (A) Hematoxylin and eosin (HE) staining of bone marrow. (B) Immunohistochemistry staining of bone marrow for cytokeratin 7 (CK7). (C) HE staining of liver tissue. (D) Immunohistochemistry staining of liver tissue for CK7.

a right ureteral tumor. The patient subsequently underwent a percutaneous liver biopsy. Immunohistochemical staining of the liver tissue, performed on January 26, 2013, showed positive results for CK7 and p63, a punctuate positive result for CD10, a weakly positive result for homeobox protein CDX-2, a suspicious result for glypican-3, and negative results for hepatocyte, Cam5.2, and Arginase (Fig. 2C and D). Meanwhile, immunohistochemistry staining of the voided urine cytology (January 22, 2013) showed positive results for CK7 and CK20, and a negative result for PSA. On January 26, 2013, the patient was diagnosed with systemic multi-site metastases from ureteral UC.

Due to the patient's poor performance status, only two cycles of intravenous gemcitabine $(1,800 \mathrm{mg}$, days 1 and 8 , every 21 days plus cisplatin (500 mg, day 1 every 21 days) were administered. The patient succumbed to disease progression 4 months later (June 2013). From diagnosis to mortality, the patient survived for $\sim 6$ months.

\section{Discussion}

The present study describes a delayed diagnosis of ureteral UC due to the lack of pathological evidence. As the patient refused to undergo endoscopic examinations again, it is unknown whether there were synchronous or metachronous ureteral tumors in other locations of the urinary system.

Ureteral UC is a rare malignant tumor. The most common clinical manifestations of ureteral UC are hematuria, increased urinary frequency, dysuria and pain. Pyuria and a palpable mass are much less frequently observed (1). Bone is one of the common metastatic sites of ureteral UC $(7,8)$. Due to the drainage through the pelvic veins into the lumbar plexus, the bone metastases of ureteral UC most commonly affect the spine (8). Although information on the type of bone destruction of ureteral UC is limited, an osteoblastic or a mixed osteolystic-osteoblastic pattern of bone destruction of transitional cell carcinoma from the bladder has been reported (9). UC should be therefore be preferentially considered as a primary tumor for osteoblastic metastasis of the spine besides prostate cancer.

However, all the typical clinical manifestations of ureteral UC were not observed in the present patient. The patient was not aware of the condition until thoracodorsal pain occurred when the disease progressed to bone metastases. The lack of pathological evidence significantly delayed the diagnosis of the primary tumor (the negative result of the first bone marrow biopsy may have been caused by an unsuitable puncture site), even though radiographic examination significantly suggested ureteral UC, and the bone metastases of the patient were osteoblastic and mainly involved the spine.

Currently, imaging and endoscopy, combined with pathological examination, are the main diagnostic approaches for UC (10-12). According to the present study, a more efficient diagnostic method is required. However, pathological evidence remains the current golden criterion for a ureteral UC diagnosis. Hence, the difficulty in achieving pathological evidence (as reported in the present study) will delay the diagnosis, no matter which diagnostic method the patient received or how efficient this was. Notably, in other tumors with the same situation (e.g., leptomeningeal metastasis), the National Comprehensive Cancer Network guidelines allow clinicians to make the diagnosis without pathological evidence (13). The guidelines offer multiple diagnostic criteria for such a unique situation. Accordingly, the possibility of new diagnostic criterion that do not rely on the pathology of primary foci should be considered in ureteral UC. 
According to the medical history of the patient, the risk of UC was high in the present study. Although there is currently insufficient evidence to recommend a screening test for the whole UC population $(14,15)$, a screening test should be recommended for the sub-population who are at high risk. Recently, UC of the bladder and the upper tract have been noted to represent two distinct diseases with practical, anatomical, biological and molecular differences (16). Hence, a screening test of UC should consider the differences between the two distinct diseases. From cytology to biomarkers, a number of novel approaches to screen UC in high-risk patients have been investigated $(17,18)$. Compared with cytology, using cost-efficient high-performing urinary biomarkers may be more beneficial in these particular patients (14). Moreover, cytology may not be appropriate to be used as a single screening test on the basis of the current study results. Hence, more attention should be aimed at the investigation of using combination strategies for the screening test in the sub-population at high risk.

In conclusion, the present study reports the case of a patient with ureteral UC presenting with osteoblastic metastases, in which the diagnosis was delayed due to the lack of symptoms and pathological evidence. Considering the possibility of asymptomatic ureteral UC and negative pathology, a suitable screening test should be recommended for high-risk patients. Additionally, a more efficient diagnostic method is required. Moreover, the possibility of new diagnostic criteria that do not rely on the pathology of primary foci in ureteral UC should be considered due to the difficulty in achieving pathological evidence in certain patients.

\section{References}

1. Bennington $\mathrm{J}$ and Beckwith JB: Tumors of the kidney, renal pelvis and ureter. In: Atlas of Tumor Pathology. 2nd series, fasc 12. Armed Forces Institute of Pathology, pp320-322, 1975.

2. Winalski CS, Lipman JC and Tumeh SS: Ureteral Neoplasms. Radiographics 10: 271-283, 1990.

3. Babjuk M, Oosterlinck W, Sylvester R, Kaasinen E, Böhle A, Palou-Redorta J and Rouprêt M; European Association of Urology (EAU): EAU guidelines on non-muscle-invasive urothelial carcinoma of the bladder, the 2011 update. Eur Urol 59: 997-1008, 2011.

4. Margulis V, Shariat SF, Matin SF, Kamat AM, Zigeuner R, Kikuchi E, Lotan Y, Weizer A, Raman JD and Wood CG; Upper Tract Urothelial Carcinoma Collaboration: Outcomes of radical nephroureterectomy: A series from the Upper Tract Urothelial Carcinoma Collaboration. Cancer 115: 1224-1233, 2009.
5. Clark PE, Spiess PE and Agarwal N, et al: Bladder Cancer: National Comprehensive Cancer Network guidelines. https://www.nccn.org/ professionals/physician_gls/f_guidelines.asp\#bladder. Accessed May 21, 2015

6. Rouprêt M1, Babjuk M, Compérat E, Zigeuner R, Sylvester RJ, Burger M, Cowan NC, Böhle A, Van Rhijn BW, Kaasinen E, et al: European Association of Urology guidelines on upper urinary tract urothelial cell carcinoma: 2015 update. Eur Urol 68: 868-879, 2015.

7. Batata MA, Whitmore WF, Hilaris BS, Tokita N and Grabstald H: Primary carcinoma of the ureter: A prognostic study. Cancer 35: 1626-1632, 1975.

8. Saitoh H, Hida M, Nakamura K and Satoh T: Distant metastasis of urothelial tumors of the renal pelvis and ureter. Tokai J Exp Clin Med 7: 355-364, 1982.

9. Goldman SM,Fajardo AA,Naraval RC and Madewell JE: Metastatic transitional cell carcinoma from the bladder: Radiographic manifestions. AJR Am J Roentgenol 132: 419-425, 1979.

10. Krabbe LM, Bagrodia A, Westerman ME and Margulis V: Diagnosis and management of upper tract urothelial carcinoma. Minerva Urol Nefrol 66: 37-48, 2014.

11. Yakoubi R, Colin P, Seisen T, Léon P, Nison L, Bozzini G, Shariat SF and Rouprêt M: Radical nephroureterectomy versus endoscopic procedures for the treatment of localised upper tract urothelial carcinoma: A meta-analysis and a systematic review of current evidence from comparative studies. Eur J Surg Oncol 40: 1629-1634, 2014

12. Raman SP and Fishman EK: Bladder malignancies on CT: The underrated role of CT in diagnosis. AJR Am J Roentgenol 203: 347-354, 2014.

13. Brem SS, Bierman PJ, Brem H, Butowski N, Chamberlain MC, Chiocca EA, DeAngelis LM, Fenstermaker RA, Friedman A, Gilbert MR, et al: Central nervous system cancers. J Natl Compr Canc Netw 9: 352-400, 2011.

14. Larre S, Catto JW, Cookson MS, Messing EM, Shariat SF, Soloway MS, Svatek RS, Lotan Y, Zlotta AR and Grossman HB: Screening for bladder cancer: Rationale, limitations, whom to target, and perspectives. Eur Urol 63: 1049-1058, 2013.

15. Xylinas E, Kluth LA, Rieken M, Karakiewicz PI, Lotan Y and Shariat SF: Urine markers for detection and surveillance of bladder cancer. Urol Oncol 32: 222-229, 2014.

16. Green DA, Rink M, Xylinas E, Matin SF, Stenzl A, Roupret M, Karakiewicz PI, Scherr DS and Shariat SF: Urothelial carcinoma of the bladder and the upper tract: Disparate twins. J Urol 189: 1214-1221, 2013.

17. Zincke H, Aguilo JJ, Farrow GM, Utz DC and Khan AU: Significance of urinary cytology in the early detection of transitional cell cancer of the upper urinary tract. J Urol 116: 781-783, 1976.

18. Ho KJ and Kuo SH: Urinary beta-glucuronidase activity as an initial screening test for urinary tract malignancy in high risk patients. Comparison with conventional urine cytologic evaluation. Cancer 76: 473-478, 1995. 